\title{
Deviations from Expectations: A Commentary on Aliev et al.
}

\author{
Sophie van der Sluis ${ }^{1,2} \cdot$ César-Reyer Vroom ${ }^{1,2} \cdot$ Conor V. Dolan $^{3}$
}

Published online: 21 February 2018

(c) The Author(s) 2018. This article is an open access publication

The Trait-based Association Test that uses Extended Simes (TATES, Van der Sluis et al. 2013) was proposed as a multivariate test in the context of genome-wide association studies (GWAS). In regular univariate GWAS, the statistical association between a phenotype of interest (e.g., height) and a single nucleotide polymorphism (SNP) is tested, yielding a single p-value. If $m$ phenotypes are studied, each phenotype can be individually regressed on the SNP, yielding $m$ p-values. TATES is a so-called combination test: it tests a multivariate hypothesis by combining the $m$ p-values obtained in the $m$ univariate tests. Specifically, TATES is based on selection of the minimal p-value among $m$ appropriately weighted p-values, and as such tests the hypothesis that at least one of the $m$ phenotypes is associated to the particular SNP. TATES was inspired by the GATES procedure, a gene based test of association ( $\mathrm{Li}$ et al. 2011).

Aliev et al. set out to demonstrate that the Type I error rate of TATES is incorrect. To this end, they present results of a small simulation study in which they examined the empirical Type I error rate of TATES given two or three correlated phenotypes, and a mathematical proof showing

This comment refers to the article available at https://doi. org/10.1007/s10519-018-9890-6.

Electronic supplementary material The online version of this article (https://doi.org/10.1007/s10519-018-9891-5) contains supplementary material, which is available to authorized users.

Sophie van der Sluis

s.vander.sluis@vu.nl

1 Department of Clinical Genetics, Section Complex Trait Genetics, Center for Neurogenomics and Cognitive Research, Amsterdam Neuroscience, VU Medical Centre Amsterdam (VUmc), Amsterdam, The Netherlands

2 Department of Complex Trait Genetics, Center for Neurogenomics and Cognitive Research, Amsterdam Neuroscience, VU University Amsterdam, De Boelelaan 1085, 1081 HV Amsterdam, The Netherlands

3 Department of Biological Psychology, VU University Amsterdam, Amsterdam, The Netherlands that the distribution of TATES p-values is not uniform under the null-hypothesis (H0) given two phenotypes.

We gratefully use this opportunity to comment on this work.

\section{Empirical Type I error rates}

In the original TATES paper, the authors showed in 20 scenarios ( 8 of which concerned the effect of missing data) that the Type I error rate of TATES is correct when the number of phenotypes $m$ equaled 20, the number of simulations $N$ sim equaled 2000, and $\alpha$ was set to 0.05 . These simulation settings were deemed realistic in the context of questionnaire data (i.e., psychological questionnaires often consist of $>10$ items, which one may want to study individually), and tailored to this context, featuring various realistic models to account for the phenotypic covariance structure (specifically, 1-, 2- or 4-factor models and network models).

Aliev et al. report simulations featuring $m=2$ and $m=3$ phenotypes in 10 correlational settings. In these simulations, 6 out of $10(m=2)$, and 21 out of $30(m=3)$ phenotypic correlations $r>0.70$ (see Aliev et al., Table 1, column 4, and Table 2, column 5). They then show that, given Nsim $=100,000$, the Type I error rate of TATES deviated significantly from 0.05 (95\% confidence interval: $\left.\mathrm{CI}_{95}=(0.04865,0.05135)\right)$ in 8 of the 10 scenarios when $m=2$, with a maximal empirical rate of 0.0553 (when $r=0.9343$ ) instead of expected 0.05 . When $m=3$, the Type I error rate of TATES deviated significantly from 0.05 in all 10 presented scenarios, with a maximal empirical rate of 0.0540 ( when $r_{1,2}=0.81, r_{1,3}=0.95, r_{2,3}=0.78$ ). Before we dwell on the statistical and practical significance of deviations of this magnitude, we first wish to gain a more comprehensive view of TATES's Type I error rate.

\section{Comprehensive simulations}

To investigate the empirical Type I error rate of TATES more extensively, we ran additional simulations in which 
we varied both the number of phenotypes $m$ and the correlations $r$ between the $m$ phenotypes. Specifically, we simulated data for $m=2,4,8$, and 16 , and $r=0.1,0.3,0.5$, 0.7 , and 0.9 , resulting in 20 simulation settings in total. Note that the resulting correlational structure is compound symmetric (i.e., all phenotypes correlated equally strong), which is consistent with a single (parallel) factor model. We simulated phenotypic and genotypic data for $N=2000$ subjects. Like Aliev et al., we simulated a single diallelic variant (unassociated, $M A F=0.5$ ), and ran Nsim $=100,000$ simulations for each setting.

To broaden the present scope of our simulations and to put the TATES results into perspective, we analyzed the simulated data using TATES and three other combination tests that, like TATES, are based on selection of the minimal p-value among $m$ weighted p-values.

The first combination test that we consider is based on Bonferroni correction (referred to as $\operatorname{minP}_{\text {Bonf }}$; Simes 1986). Running $m$ univariate analyses to regress $m$ phenotypes on a SNP, the $m$ p-values are all Bonferronicorrected (i.e., weighted with $m$ ), and then the smallest Bonferroni-corrected p-value is selected. The second combination test, which we refer to as $\operatorname{minP}_{\mathrm{NS}}$, is similar to $\min _{\text {Bonf }}$, except that one does not correct for the observed number of phenotypes $m$, but for the effective number of phenotypes $M_{\text {eff }}$. As suggested by Nyholt (2004, based on Šidák 1968, 1971), we calculated $M_{\text {eff }}$ based on eigenvalue decomposition of the $m \times m$ phenotypic correlation matrix, and the smallest weighted p-value is selected as $\operatorname{minP}_{\mathrm{NS}}$ p-value. Note that, assuming non-zero phenotypic correlations, $M_{\text {eff }}$ is always $<m$, and $\operatorname{minP}_{\mathrm{NS}}$ is thus always less strict than $\operatorname{minP}_{\text {Bonf }}$.

The third combination test is the original Simes test that TATES is a variation on (Simes 1986). In Simes, the $m$ p-values are first sorted ascendingly. In an iterative fashion, each $j$ th $\mathrm{p}$-value of the $m$ sorted $\mathrm{p}$-values is then weighted with $m / j$, such that the lowest p-value is weighted with the largest weight (i.e., $\mathrm{m} / \mathrm{l}$ ) and the highest $\mathrm{p}$-value is weighted with the smallest weight (i.e., $m / m=1$ ). The Simes p-value then corresponds to the smallest weighted p-value.

TATES, which is based on GATES (Li et al. 2011), weights in fashion similar to Simes, except that the observed number of p-values $m$ and $j$ are replaced with the effective number of p-values $m_{\mathrm{e}}$ and $m_{\mathrm{ej}}$. Specifically, TATES weights each $j$ th $\mathrm{p}$-value $p_{\mathrm{j}}$ by $m_{\mathrm{e}} / m_{\mathrm{ej}}$, and $m_{\mathrm{ej}}$ is calculated as

$m_{e j}=j-\sum_{i=1}^{j} I\left(\lambda_{i}>1\right)\left(\lambda_{i}-1\right)$

where $j$ is the number of top $j$ p-values, $\lambda_{\mathrm{i}}$ denotes the ith eigenvalue of the correlation matrix between the $j$ p-values (which can be approximated from the correlations between the $j$ phenotypes), and $\mathrm{I}(\mathrm{x})$ is an indicator function taking on value 0 if $\lambda_{\mathrm{i}} \leq 1$ and 1 if $\lambda_{\mathrm{i}}>1$. That is, the effective number of p-values $m_{\mathrm{ej}}$ among the $j \mathrm{p}$-values is calculated as the observed number of $\mathrm{p}$-values $j$ minus the sum of the difference between the eigenvalues $\lambda_{i}$ and 1 for those eigenvalues $\lambda \mathrm{i}>1$. The value of $m_{\mathrm{e}}$ is equal to $m_{\mathrm{ej}}$ for the case that $j=m$, i.e., when the selection of top p-values covers all p-values. The TATES p-value then corresponds to the smallest weighted p-value. Following this procedure, the smallest original p-value is always weighted by the largest weight, while the largest original p-value is weighted by $m_{\mathrm{e}} / m_{\mathrm{e}}=1$ as in that case $m_{\mathrm{ej}}=m_{\mathrm{e}}$. As the weight $m_{\mathrm{e}} / m_{\mathrm{ej}}$ is always $\geq 1$, the weighted p-values are, like in the Simes test, always $\geq$ the original, unweighted p-values.

All four combination tests test the hypothesis that at least 1 of the $m$ phenotypes is associated to the SNP under study by assessing whether the selected weighted p-value is smaller than a beforehand established threshold (it being 0.05 , or the default genome-wide threshold $5 \times 10^{-8}$ ).

We ran the 20 simulation scenarios for all four methods, and then established the percentage of p-values per scenario smaller than 0.05 . For all four methods, these observed Type I error rates are shown in Table 1. We then established whether the observed percentage fell inside the $\mathrm{CI}_{95}$ given $\alpha=0.05$. The standard error of the ML estimator of the p-value is $S E=\sqrt{p \times(1-p) / N \operatorname{sim}}$, where $p$ denotes the percentage of significant tests expected given the chosen $\alpha$ (i.e., 0.05), and Nsim the total number of simulations. Given Nsim $=100,000$ and $\alpha=0.05$, the $\mathrm{CI}_{95}$ thus equals $(p-1.96 \times \mathrm{SE}, p+1.96 \times \mathrm{SE})=(0.04865$, $0.05135)$. In Table 1 , values that fall outside the $\mathrm{CI}_{95}$ given Nsim $=100,000$ are italicized, while values falling outside the $\mathrm{CI}_{95}$ given $N$ sim $=10,000$ are italicized and bold.

The Type I error results in Table 1 show that when correlations are medium-to-high, TATES is slightly liberal when $m$ is small, yet slightly conservative when $m$ is large. Simes and $\min \mathrm{P}_{\mathrm{Bonf}}$ are almost always conservative, and becomes more so with increasing correlations and increasing $m$. In contrast, $\min _{\mathrm{NS}}$ is generally liberal, and especially so when $m$ is small and correlations are high. If we sum the absolute deviations from 0.05 across all 20 scenarios, we see that overall, TATES remains closest to 0.05 , while $\operatorname{minP}_{\mathrm{Bonf}}$ shows the largest overall deviation, entirely due to its conservativeness. Indeed, $\operatorname{minP}_{\text {Bonf }}$ shows the largest undershoot, while $\min _{\mathrm{NS}}$ shows the largest overshoot.

Overall, the Type I error rate of TATES does show $m$ - and $r$-dependent variation around 0.05 , but these deviations are small, especially compared to the other considered combination tests. The deviations reported by Aliev et al. are associated with the special case of small $m$ and (very) high $r$. However, regardless of the narrow scope of the simulations by Aliev et al., one may still ask whether the observed deviations are a reason to reject the TATES procedure. 
Table 1 Type I error rates of four combination tests in 20 simulation scenarios

\begin{tabular}{|c|c|c|c|c|}
\hline Correlations & TATES & Simes & $\min P_{\text {Bonf }}$ & $\min _{\mathrm{NS}}$ \\
\hline \multicolumn{5}{|l|}{ Nvar $=2$} \\
\hline 0.1 & 0.05012 & 0.05006 & 0.04932 & 0.04955 \\
\hline 0.3 & 0.05009 & 0.04887 & 0.04804 & 0.05033 \\
\hline 0.5 & 0.05230 & 0.04858 & 0.04704 & 0.05326 \\
\hline 0.7 & 0.05172 & 0.04408 & 0.04153 & 0.05459 \\
\hline 0.9 & 0.05550 & 0.04191 & 0.03672 & 0.05967 \\
\hline \multicolumn{5}{|l|}{ Nvar $=4$} \\
\hline 0.1 & 0.05000 & 0.04987 & 0.04890 & 0.04925 \\
\hline 0.3 & 0.05148 & 0.04976 & 0.04807 & 0.05123 \\
\hline 0.5 & 0.05128 & 0.04579 & 0.04291 & 0.05268 \\
\hline 0.7 & 0.05115 & 0.04089 & 0.03637 & 0.05583 \\
\hline 0.9 & 0.05324 & 0.03511 & 0.02654 & 0.06250 \\
\hline \multicolumn{5}{|l|}{ Nvar $=8$} \\
\hline 0.1 & 0.05025 & 0.04999 & 0.04883 & 0.04930 \\
\hline 0.3 & 0.05005 & 0.04777 & 0.04579 & 0.04955 \\
\hline 0.5 & 0.04924 & 0.04350 & 0.03968 & 0.04949 \\
\hline 0.7 & 0.04742 & 0.03699 & 0.03135 & 0.05175 \\
\hline 0.9 & 0.04495 & 0.02881 & 0.01821 & 0.05524 \\
\hline \multicolumn{5}{|l|}{ Nvar $=16$} \\
\hline 0.1 & 0.04977 & 0.04945 & 0.04803 & 0.04851 \\
\hline 0.3 & 0.04891 & 0.04659 & 0.04363 & 0.04773 \\
\hline 0.5 & 0.04674 & 0.04110 & 0.03651 & 0.04664 \\
\hline 0.7 & 0.04093 & 0.03209 & 0.02576 & 0.04408 \\
\hline 0.9 & 0.03677 & 0.02502 & 0.01314 & 0.04660 \\
\hline Mean (SD) & $0.0491(0.0042)$ & $0.0428(0.0076)$ & $0.0388(0.0108)$ & $0.0514(0.0045)$ \\
\hline Largest overshoot & 0.0055 & 0 & 0 & 0.0125 \\
\hline Largest undershoot & 0.0132 & 0.0250 & 0.0369 & 0.0059 \\
\hline $\begin{array}{l}\text { Sum of absolute deviations } \\
\text { across all conditions }\end{array}$ & 0.0525 & 0.1439 & 0.2236 & 0.0664 \\
\hline
\end{tabular}

Italicized values lie outside the $95 \%$ confidence interval given Nsim $=100,000\left(\mathrm{CI}_{95}=0.0486-0.0514\right)$. Italicized and bold values lie outside the $95 \%$ confidence interval given $\mathrm{Nsim}=10,000\left(\mathrm{CI}_{95}=0.0457-\right.$ $0.0543)$.

\section{Power to detect departures from nominal a}

The larger the number of replications in a simulation study, the more power one has to demonstrate that the empirical Type I error rate of a method deviates from the expected Type I error rate. For instance, with Nsim $=2000$ (original TATES paper), Nsim $=10,000$, and Nsim $=100,000$ (Aliev et al., and present simulations), the $\mathrm{CI}_{95}$ 's of an unbiased p-value are 0.0404-0.0596, 0.0457-0.0543, and 0.0486-0.0514, respectively. The empirical Type I error rates of TATES, as displayed in Table 1, should thus be considered incorrect in either 1,3 , or, 10 of the 20 scenarios, depending on the power, i.e., the chosen Nsim. Aliev et al. emphasized statistical significance in assessing the Type I error results. However, in this situation, we believe that it is more important to consider the practical relevance of deviations of $0.05,0.005$, or 0.001 . We are convinced that deviations of this magnitude, while statistically significant given large Nsim, cannot justify the rejection of TATES, or any other method, and believe therefore that the TATES Type I error rates give little reason for concern.

\section{The assumption of uniformly distributed p-values}

Aliev et al. argued that the distribution of the TATES p-values should be uniform under the $\mathrm{H} 0$, and that the probability distribution function should at least not exceed 1 at the low end of the distribution, i.e., around 0 . They consider this a condition for the Type I error rate of TATES to be correct. It is important to note that p-values may not be uniformly distributed under the $\mathrm{H} 0$ in special cases (see Aliev et al.'s references to Murdoch et al. 2008; Bland 2013). However, in most statistical tests, when distributional assumptions and sample size requirements are met, the resulting $\mathrm{p}$-values 
are indeed assumed to be uniformly distributed under $\mathrm{H} 0$. Combination tests like $\operatorname{minP}_{\mathrm{Bonf}}, \mathrm{minP}_{\mathrm{NS}}$, and TATES work with these $\mathrm{p}$-values. However, these combination tests themselves are based on selection of the minimal p-value among $m$ weighted p-values. So while the p-values of the $m$ univariate tests, on which these combination tests are based, should indeed generally be uniformly distributed, the relevant question here is whether the p-values resulting from
A

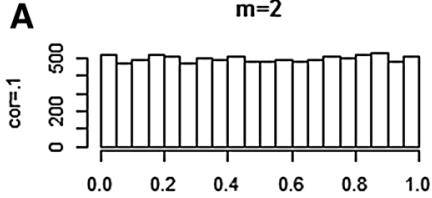

售

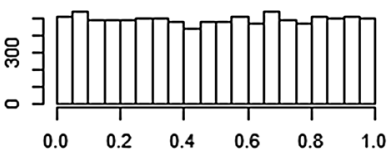

芒

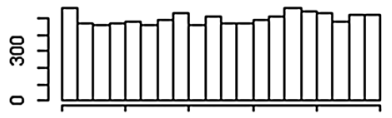

농
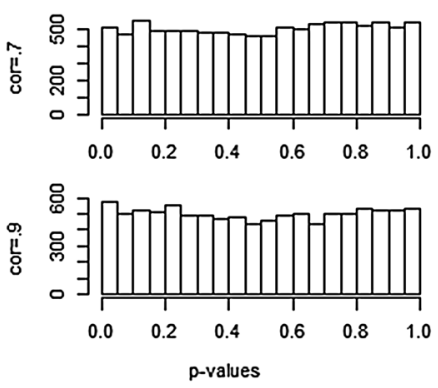

B

$\mathrm{m}=2$
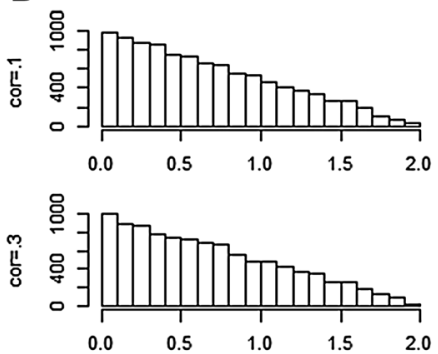

过
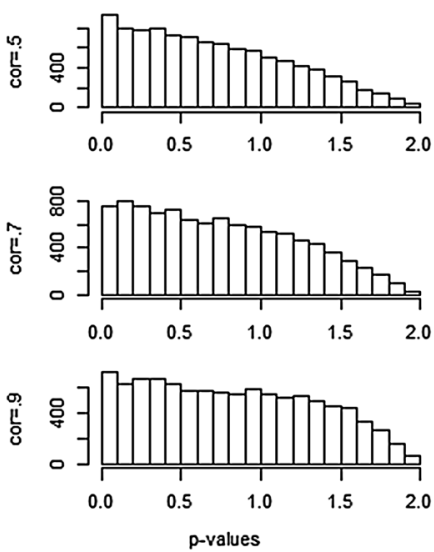

$\mathrm{m}=4$
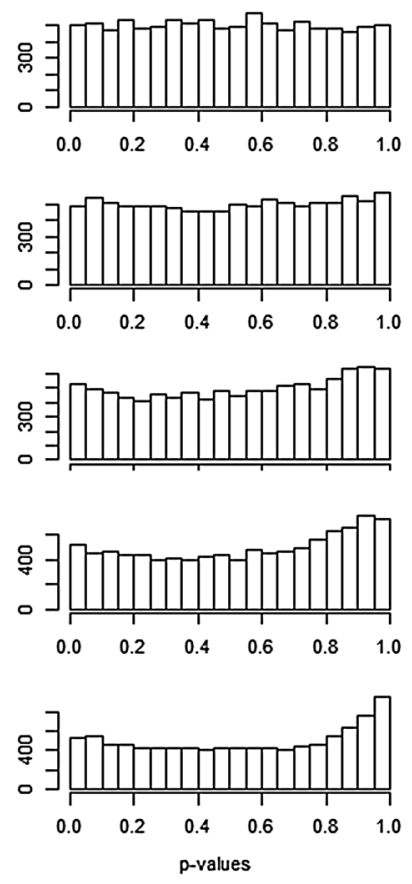

$m=4$
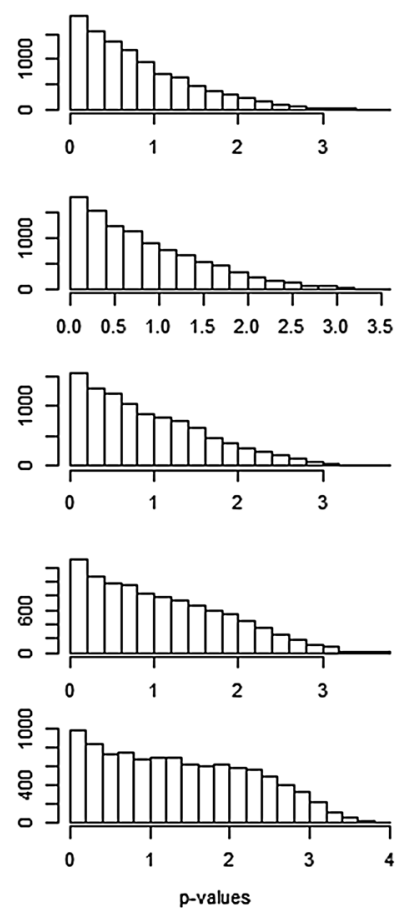

Fig. 1 P-value distributions under the H0 for TATES (a) and minP $\mathrm{B}_{\mathrm{Bonf}}$ (b) in 20 simulated scenarios varying the number of phenotypes $m$ (columns) and the correlations between these phenotypes (rows).
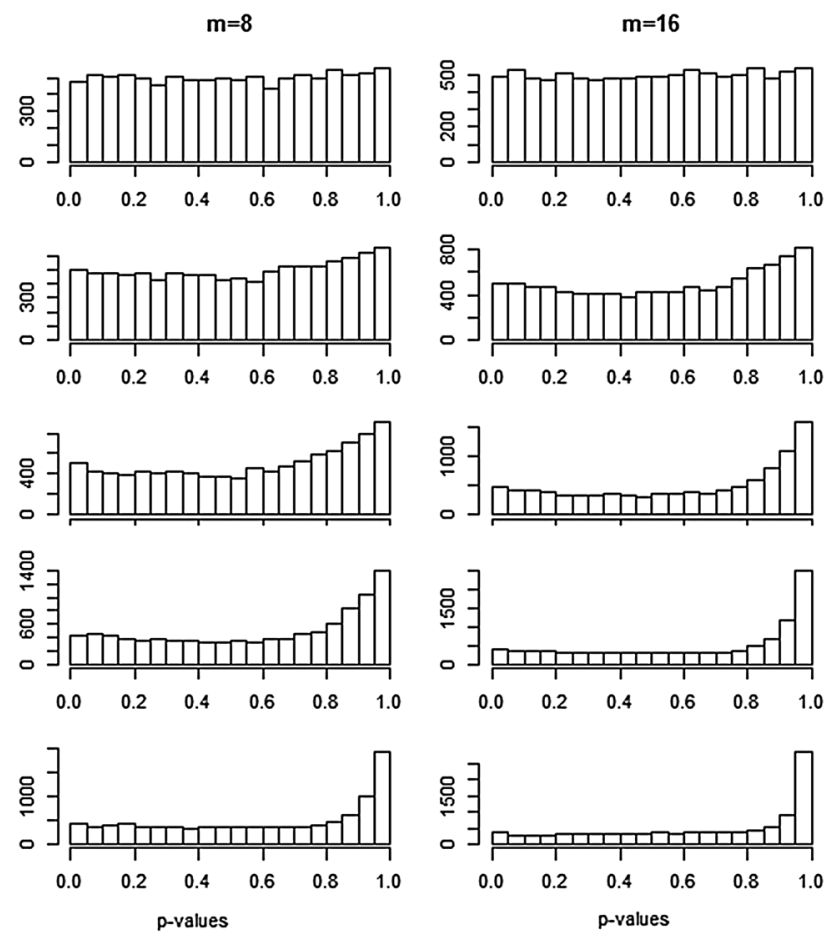

$\mathrm{m}=8$
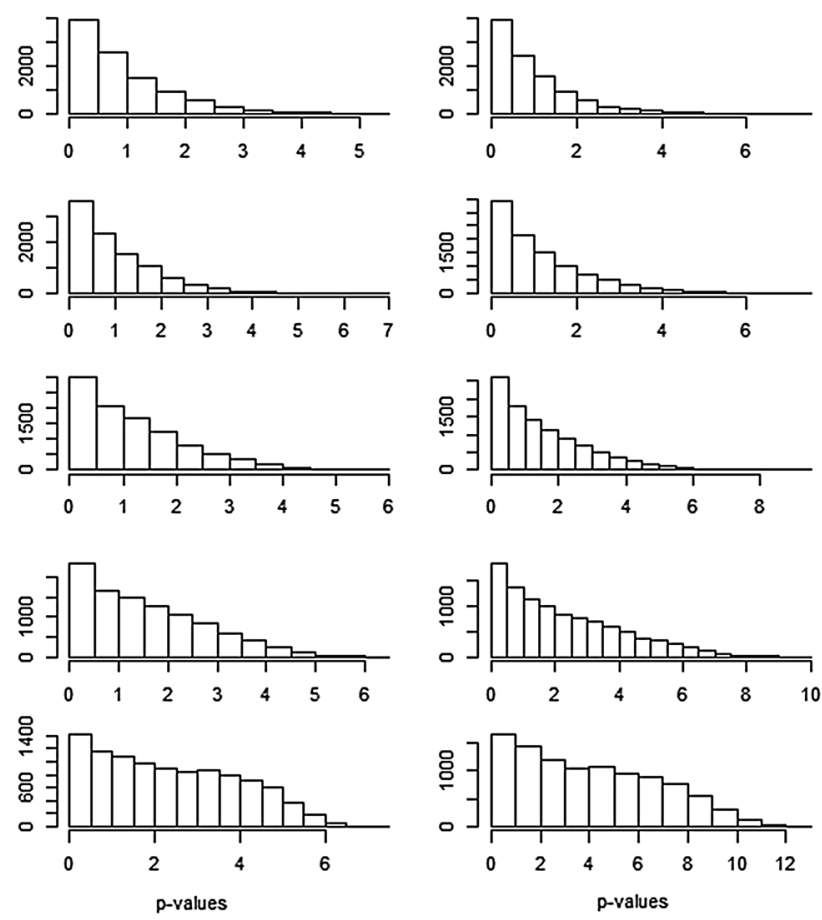

Note that the p-value distributions of Simes and $\operatorname{minP}_{\mathrm{NS}}$ are quite similar to those of TATES and $\mathrm{minP}_{\mathrm{Bonf}}$, respectively, and are therefore not displayed separately 
combination tests' weighted-selection procedure should be uniformly distributed as well.

Before we discuss the distributions of the p-values of these four combination tests for the 20 simulation scenarios described above, we wish to emphasize the nature of the weighting in the four different tests. In $\operatorname{minP}_{\mathrm{Bonf}}$ and $\operatorname{minP}_{\mathrm{NS}}$, all $m$ p-values are weighted by the same constant, being $m$ for $\min \mathrm{P}_{\mathrm{Bonf}}$ and $M_{\mathrm{eff}}$ for $\operatorname{minP}_{\mathrm{NS}}$. In the original Simes test and TATES, however, each of the $m$ p-values is weighted differently, i.e., each $j$ th $\mathrm{p}$-value among the $m$ ascendingly sorted p-values is weighted with $m / j$ or $m_{\mathrm{e}} / m_{\mathrm{ej}}$, respectively.

Figure 1 shows the distributions of the first 10,000 (of $100,000) \mathrm{p}$-values obtained with TATES and $\operatorname{minP}_{\text {Bonf }}$ (panel $a$ and $b$, respectively) in each of the 20 aforementioned simulation settings (the p-value distributions obtained with Simes and $\operatorname{minP}_{\mathrm{NS}}$ are very similar to those of TATES and $\min _{\mathrm{Bonf}}$, respectively, and therefore not shown separately).

Clearly, $p$-value distributions of methods that are based on selection of the minimal weighted $p$-value are not uniformly distributed under $\mathrm{H} 0$, even if the $m$ p-values that they are based on are. Specifically, when all p-values are weighted with the same weight $\left(\operatorname{minP}_{\mathrm{Bonf}}, \min _{\mathrm{NS}}\right)$, selection of the minimal weighted $\mathrm{p}$-value results (as could be expected) in a p-value distribution with a right, positive skew. When smaller p-values are weighted more heavily, the deviation of uniformity increases with increasing $m$ and increasing correlations, with the bulk of p-values at the high end of the distribution. Indeed, as both Simes and TATES weight the highest original p-value with the smallest weight (i.e., 1 , see above), the Simes p-value and the TATES p-value very often equal the largest $\mathrm{p}$-value before weighting.

\section{Conclusion}

Aliev et al. set out to show that the Type I error rate of TATES procedure is incorrect by presenting results of a simulation study and a mathematical proof concerning the nonuniformity of TATES's p-value distribution. With respect to the former, we believe that the simulation results of Aliev et al. as well as our own showed that TATES's Type I error rate indeed shows slight in- or deflation, depending on the number of phenotypes $m$ and the strength of their intercorrelations $r$. However, we consider the observed deviations of 0.05 , whether or not statistically significant, to be too small to be considered of practical concern. Note that in this commentary, we addressed the Type I error rate given an expected rate of $\alpha=0.05$, like Aliev et al. did. We also considered $\alpha=0.01$ and $\alpha=0.001$ (Supplemental Table 1) and found that Type I error rates of TATES were close to expectation (ranges 0.0081-0.0120, and 0.00094-0.00129, respectively). While again some values deviated statistically significantly from expectation (specifically, given $\alpha=0.01$ and $\alpha=0.001,1$ and 0 values of the 20 simulated settings fell outside the $\mathrm{CI}_{95}$ given $N$ sim $=10,000$, and 9 and 5 fell outside the $\mathrm{CI}_{95}$ given $N \operatorname{sim}=100,000$, respectively), the deviations were small and, in our view, of little practical significance.

With respect to mathematical proof concerning the uniformity of the p-value distribution, we believe that the assumption that the distribution of $\mathrm{p}$-values of a method that is based on p-value selection should be uniform, is based on misconception. The p-values that the combination tests are based on should be uniformly distributed, but the p-values of subsequent weighted-selection-based combination tests are not expected to be uniformly distributed.

All in all, if one wishes to apply a combination test to tackle a multivariate problem, we believe that TATES represents a viable option.

Acknowledgements This work was funded by The Netherlands Organization for Scientific Research (NWO MagW VIDI 452-12-014).

\section{Compliance with Ethical Standards}

Conflict of interest Sophie van der Sluis, César-Reyer Vroom and Conor V. Dolan declare that they have no conflict of interest.

Human and Animal Rights This article does not contain any studies with human participants or animals performed by any of the authors.

Informed Consent Only simulated data were used in this study.

Open Access This article is distributed under the terms of the Creative Commons Attribution 4.0 International License (http://creativeco mmons.org/licenses/by/4.0/), which permits unrestricted use, distribution, and reproduction in any medium, provided you give appropriate credit to the original author(s) and the source, provide a link to the Creative Commons license, and indicate if changes were made.

\section{References}

Bland M (2013) Do baseline P-values follow a uniform distribution in randomised trials? PLoS ONE 8(10):1-5

Li M-X, Gui H-S, Kwan JSH, Sham PC (2011) GATES: a rapid and powerful gene-based association test using extended Simes procedure. Am J Hum Genet 88:283-293

Murdoch DJ, Tsai Y-L, Adcock J (2008) P-Values are random variables. Am Stat 62(3):242-245

Nyholt DR (2004) A simple correction for multiple testing for singlenucleotide polymorphisms in linkage disequilibrium with each other. Am J Hum Genet 74:765-769

Šidák Z (1968) On multivariate normal probabilities of rectangles: their dependence on correlations. Ann Math Statist 39:1425-1434

Šidák Z (1971) On probabilities of rectangles in multivariate normal Student distributions: their dependence on correlations. Ann Math Statist 41:169-175

Simes RT (1986) An improved Bonferroni procedure for multiple tests of significance. Biometrika 73(3):751-754

Van der Sluis S, Posthuma D, Dolan CV (2013) TATES: Efficient multivariate genotype-phenotype analysis for genome-wide association studies. PLoS Genet 9(1):1-9 